\title{
Optimal Active Target Localisation Strategy with Range-Only Measurements
}

\author{
Shaoming He ${ }^{\circledR a}$, Hyo-Sang Shin $\circledast^{\mathrm{b}}$ and Antonios Tsourdos $\mathbb{1}^{\mathrm{c}}$ \\ School of Aerospace, Transport and Manufacturing, Cranfield University, College Road, Cranfield, MK43 OAL, UK \\ \{shaoming.he, h.shin, a.tsourdos\}@cranfield.ac.uk
}

\begin{abstract}
Keywords: Target localisation, Range-only measurement, Optimal manoeuvre
Abstract: $\quad$ This paper investigates the problem of one-step ahead optimal active sensing strategy to minimise estimation errors with range-only measurements for non-manoeuvring target. The determinant of Fisher Information Matrix (FIM) is utilized as the objective function in the proposed optimisation problem since it quantifies the volume of uncertainty ellipsoid of any efficient estimator. In consideration of physical velocity and turning rate constraints, the optimal heading angle command that maximises the cost function is derived analytically. Simulations are conducted to validate the analytical findings.
\end{abstract}

\section{INTRODUCTION}

The past few years have witnessed an increasing interest in the development and employment of small-scale unmanned robots in both civil and military applications. One particular interesting mission of smallscale unmanned robots is to track and localize targets of interest in an automated fashion since reliable target tracking is a fundamental and key enabling technology in many practical applications, such as vehicle navigation, situational awareness and public surveillance (Atanasov et al., 2014; Salaris et al., 2017). One main challenge for the operations of these smallscale robots in target localisation is that they are typically constrained by limited payload, power and endurance. Therefore, only limited information, e.g., bearing-only or range-only, can be gathered due to the limits of sensor availability.

It is well-known that the relative geometry between the observer and the target poses great effects on the achievable localisation performance (Bishop et al., 2010). For this reason, active sensing that finds the optimal path or trajectory for information gain maximisation can yield significant benefits to improving the perceptual results in target localisation (Bajcsy et al., 2018). Through numerically maximising the determinant of FIM over a finite horizon, optimal observer trajectory for bearing measurements gathering

\footnotetext{
a (iD) https://orcid.org/0000-0001-6432-5187

b (iD) https://orcid.org/0000-0001-9938-0370

c (D) https://orcid.org/0000-0002-3966-7633
}

to localize a single target was proposed in Tokekar et al. (2011). The rationale of leveraging FIM lies in that it prescribes a lower bound of target localisation error covariance of any efficient estimation algorithm (Taylor, 1979). Therefore, the determinant of FIM can be utilized as a performance metric to quantify the volume of the error uncertainty ellipsoid. Except for FIM, the trace of error covariance, which directly quantifies the average estimation performance, was also utilized to find the proper path for target localisation using bearing-only measurements (Logothetis et al., 1997; Zhou et al., 2011). Note that most approaches utilize numerical methods to find the optimal solutions and consequently require high computational burden. To alleviate the computational issue, an analytical solution was proposed in (He et al., 2019) using geometric analysis for bearing-only target localisation.

Apart from bearing-only-based target localisation, range-only-based active sensing strategy is another emerging low-cost solution for target localisation using small-scale robots due to the proliferation of lightweight and low-cost LIDAR/infrared range finders. MartíNez and Bullo (2006); Bishop et al. (2010) analysed the optimal relative target-observer geometric, that maximises system observability, for multiple static sensors to localize a stationary target, where the determinant of FIM was utilized as the cost function. A range-only-based sliding mode controller was proposed in Matveev et al. (2011) to drive a wheeled mobile robot to a predefined distance from a manoeuvring target and makes the robot follow the target at 
this distance. A similar problem was considered in Matveev et al. (2016), but this reference additional addressed the issue of turning rate limit pertaining to the robot, thus providing more realistic results. As an extension of Matveev et al. (2016), a three-dimensional circumnavigating algorithm for multiple moving targets was proposed in Matveev and Semakova (2017). In a GPS-denied environment when only range measurement is available, Cao (2015) suggested a circumnavigating algorithm to improve active perceptual results. Although these range-only-based circumnavigating algorithms show good performance in both simulations and experiments, they fail to maximise or minimise a meaningful performance measure, which is of paramount importance in active sensing. Using simplified numerical search, Yang et al. (2014) proposed an optimal sensor coordination strategy for active target localisation with range-only measurements. Although numerical optimisation methods provide exact solution in active sensing, these algorithms might not be suitable for low-cost robots due to limited computational power. For this reason, it is meaningful to derive analytically optimal solutions to improve the estimation performance.

This paper aims to to develop an analytically optimal active sensing strategy in consideration of physical constraints for non-manoeuvring target localisation with range-only measurements. This work is an extension of our previous results (He et al., 2019) to range-only scenarios. Similar to previous studies, the determinant of FIM is leveraged as the cost function in the optimisation problem. As one of the main contributions, this paper derives a closed-form solution that maximises the cost function. Furthermore, physical constraints such as minimum turning rate and velocity limits are also considered in deriving the optimal solution to support practical applications. The resultant analytical solution, given as heading angle input command, is simple to be implemented in practice. Theoretical observability analysis is also performed to support the proposed localisation algorithm.

\section{PROBLEM FORMULATION}

This section provides some necessary preliminaries of vehicle kinematics model and range-only measurement model to facilitate the analysis in the following sections. The problem formulation of this paper is also stated.

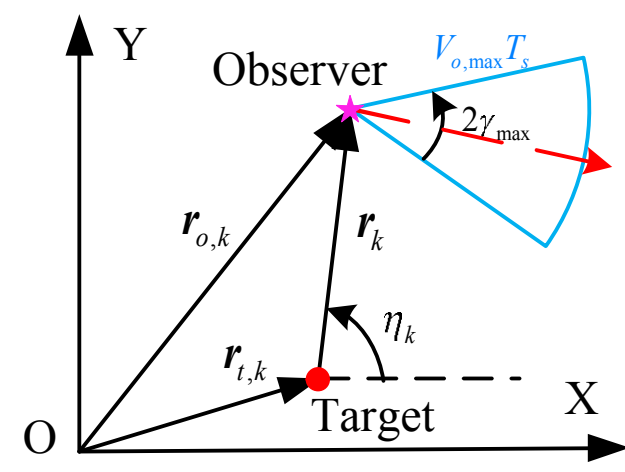

Figure 1: Geometric relationship between the observer and the target at time step $k$ in an inertial coordinate.

\subsection{Vehicle Kinematics}

This work assumes that the observer is equipped with a high-performance low-level control system that provides velocity tracking, heading and altitude hold functions. This study aims to design guidance input to this low-level controller for target localisation and only concerns the two-dimensional (2D) motions. The vehicle's kinematics in a $2 \mathrm{D}$ environment is given by

$$
\begin{aligned}
& \dot{x}_{o}=V_{o} \cos \gamma_{o} \\
& \dot{y}_{o}=V_{o} \sin \gamma_{o}
\end{aligned}
$$

where $\left(x_{o}, y_{o}\right)$ stands for the observer's position in an inertial coordinate. $\gamma_{o}$ is the observer's heading angle and $V_{o} \in\left(0, V_{o \text {,max }}\right]$ denotes the observer's velocity with $V_{o \text {,max }}$ being the maximum permissible velocity. Note that the observer's velocity $V_{o}$ is assumed to be larger than the target's velocity.

In practice, the observer heading change between two consecutive time steps is constrained due to physical turning rate limitation as

$$
\left|\gamma_{o, k}-\gamma_{o, k-1}\right| \leq \gamma_{\max } \triangleq \omega_{\max } T_{s}
$$

where $\gamma_{o, k}$ represents the heading angle at time step $k, \omega_{\max }$ the maximum permissible turning rate of the observer, and $T_{S}$ the sampling time.

\subsection{Measurement Model}

Figure 1 shows the geometric relationship between the observer and the target at time step $k$, where the observer is represented by a magenta pentagram and the red circle denotes the target. The red vector stands for the observer heading direction at previous time step. $X-O-Y$ is the inertial coordinate. The notations $\mathbf{r}_{o, k}=\left[x_{o, k}, y_{o, k}\right]^{T}$ and $\mathbf{r}_{t, k}=\left[x_{t, k}, y_{t, k}\right]^{T}$ represent the position vectors of the observer and the target at time step $k$, respectively. $\mathbf{r}_{k}=\mathbf{r}_{o, k}-\mathbf{r}_{t, k}$ denotes the relative position vector between the observer 
and the target. $\eta_{k}$ denotes the bearing angle at current time step, which represents the direction of the relative position vector. With heading constraint (2), the maximum region the observer can travel at current time step is described by the blue sector with radius $V_{o, \max } T_{s}$ and angle $2 \gamma_{\max }$, as shown in Fig. 1 .

At time step $k$, the observer only has access to the relative range provided by a range finder. Therefore, the sensor measurement $z_{k}$ is given by

$$
z_{k}=\left\|\mathbf{r}_{k}\right\|+v_{k}=\sqrt{x_{k}^{2}+y_{k}^{2}}+v_{k}
$$

where $v_{k}$ denotes the sensor measurement noise, which is assumed to be Gaussian white as $v_{k} \sim$ $\mathcal{N}\left(0, \sigma_{r}^{2}\right)$ with $\sigma_{r}$ being the standard deviation of the measurement noise.

\subsection{Problem Formulation}

The objective of this paper is to analytically find an one-step ahead optimal sensing strategy that minimises non-manoeuvring target localisation errors with range-only measurements. For this optimisation problem, we utilise the well-known FIM to formulate the cost function since the inverse of FIM prescribes a lower bound of the estimation error covariance of any efficient estimator. Assume that the relative position vector $\mathbf{r}_{k}$ is known for finding the optimal observer manoeuvre vector at time instant $k$. Then, the onestep ahead position-related FIM for range-only localisation is given by

$$
\begin{aligned}
\text { FIM } & =\frac{1}{\sigma_{r}^{2}} \sum_{i=k}^{k+1}\left[\begin{array}{ll}
\left(\frac{\partial\left\|r_{i}\right\|}{\partial x_{t, i}}\right)^{2} & \frac{\partial\left\|r_{i}\right\|}{\partial x_{t, i}} \frac{\partial\left\|r_{i}\right\|}{\partial y_{t, i}} \\
\frac{\partial\left\|r_{i}\right\|}{\partial x_{t, i}} \frac{\partial\left\|r_{i}\right\|}{\partial y_{t, i}} & \left(\frac{\partial\left\|r_{i}\right\|}{\partial y_{t, i}}\right)^{2}
\end{array}\right] \\
& =\frac{1}{\sigma_{r}^{2}} \sum_{i=k}^{k+1}\left[\begin{array}{ll}
\cos ^{2} \eta_{i} & \frac{\sin \left(2 \eta_{i}\right)}{2} \\
\frac{\sin \left(2 \eta_{i}\right)}{2} & \sin ^{2} \eta_{i}
\end{array}\right]
\end{aligned}
$$

For computational simplicity, we consider the determinant of FIM, also known as D-optimality criterion, as the cost function in our problem. The determinant of FIM quantifies the volume of the estimation error uncertainty ellipsoid and can be readily obtained from Eq. (4) as

$$
\operatorname{det}(\mathbf{F I M})=\frac{1}{\sigma_{r}^{4}} \sin ^{2}\left(\eta_{k+1}-\eta_{k}\right)
$$

Denote $\mathbf{v}_{o, k}=\left[V_{o, k} T_{S} \cos \gamma_{o, k}, V_{o, k} T_{s} \sin \gamma_{o, k}\right]^{T}$ as the observer manoeuvre vector at time step $k$ and $\sigma=$ $\eta_{k+1}-\eta_{k}$. This paper formulates the following constrained discrete-time optimisation problem, denoted as $\mathbf{C D O}_{1}$ : find the observer manoeuvre vector at time step $k, \mathbf{v}_{o, k}$, which maximises the following objective function $J$

$$
J=\sin ^{2} \sigma
$$

subject to

$$
\begin{aligned}
& \left|\gamma_{o, k}-\gamma_{o, k-1}\right| \leq \omega_{\max } T_{s} \\
& 0<V_{o, k} \leq V_{o, \max }
\end{aligned}
$$

The aim of this paper is to derive the closed-form solution of $\mathbf{C D O}_{1}$.

\section{Derivation of Optimal Sensing Strategy for Active Target localisation}

This section will propose an analytical optimal manoeuvre that maximises the cost function $J$ for target localisation with range-only measurement. We first derive the optimal solution without heading constraint and then extend to the case that the robot has limited turning rate to change its heading angle.

\subsection{Optimal Solution without Heading Constraints}

Excluding the heading constraint, $\mathbf{C D O}_{1}$ reduces to $\mathbf{C D O}_{2}$ : find the observer manoeuvre vector at time step $k, \mathbf{v}_{o, k}$, which maximises the following objective function $J$

$$
J=\sin ^{2} \sigma
$$

subject to

$$
0<V_{o, k} \leq V_{o, \max }
$$

Change in the vehicle's velocity over a short interval, like over $T_{S}$, is usually negligible. Hence, for simplicity, it is assumed that the observer moves with constant speed and constant direction between two consecutive time steps. Let $\mathbf{v}_{t, k}=\left[v_{t x, k}, v_{t y, k}\right]^{T} \triangleq$ $\left[V_{t, k} T_{s} \cos \gamma_{t, k}, V_{t, k} T_{s} \sin \gamma_{t, k}\right]^{T}$ be the target manoeuvre vector at time step $k$. Assume that the target position and velocity vector at current time step can be obtained from Kalman filter, $\mathbf{v}_{t, k}$ is known in trajectory optimisation. Note that this assumption will be validated by a detailed observability analysis provided in the next section. Figure 2 shows the geometric relationship between the observer and the target within two consecutive time steps, where $\delta \mathbf{r}_{k}$ represents the relative manoeuvre at time step $k$ and $\overline{\mathbf{r}}_{k}=\mathbf{r}_{k}-\mathbf{v}_{t, k}$ is an auxiliary vector utilised in the analysis. Since $\mathbf{v}_{t, k}$ is fixed, $\overline{\mathbf{r}}_{k}$ is known in trajectory optimisation. The analytical solution of $\mathbf{C D O}_{2}$ can be obtained using Lemmas 1 through 2.

Lemma 1. Given the observer velocity $V_{o, k}$, the candidate optimal heading angle at time step $k$ without any constraint is given by

$$
\gamma_{o, k}^{*, 1}=\arcsin \left(-\frac{V_{o, k} T_{s}}{\left\|\overline{\mathbf{r}}_{k}\right\|}\right)-\vartheta
$$




$$
\gamma_{o, k}^{*, 2}=\pi-\arcsin \left(-\frac{V_{o, k} T_{s}}{\left\|\overline{\mathbf{r}}_{k}\right\|}\right)-\vartheta
$$

with $\sin \vartheta=b / \sqrt{a^{2}+b^{2}}$ and $\cos \vartheta=a / \sqrt{a^{2}+b^{2}}$, where $a=y_{k}-v_{t y, k}, b=x_{k}-v_{t x, k}$.

Proof. For moving target, the relative manoeuvre vector at time step $k$ can be obtained as

$$
\begin{aligned}
& \delta \mathbf{r}_{k}=\mathbf{v}_{o, k}-\mathbf{v}_{t, k} \\
& =\left[V_{o, k} T_{s} \cos \gamma_{o, k}-v_{t x, k}, V_{o, k} T_{s} \sin \gamma_{o, k}-v_{t y, k}\right]^{T}
\end{aligned}
$$

Then, the relative position vector at time step $k+1$ is given by

$\mathbf{r}_{k+1}=\mathbf{r}_{k}+\delta \mathbf{r}_{k}$

$=\left[x_{k}+V_{o, k} T_{s} \cos \gamma_{o, k}-v_{t x, k}, y_{k}+V_{o, k} T_{s} \sin \gamma_{o, k}-v_{t y, k}\right]$
From Fig. 2, the separation angle $\sigma$ between two consecutive relative position vectors can be obtained as

$$
\sigma=\arccos \left(\frac{\mathbf{r}_{k}^{T} \cdot \mathbf{r}_{k+1}}{\left\|\mathbf{r}_{k}\right\|\left\|\mathbf{r}_{k+1}\right\|}\right)
$$

Substituting Eq. (14) into Eq. (6) gives

$$
J=1-\cos ^{2} \sigma=\frac{\left(x_{k} y_{k+1}-x_{k+1} y_{k}\right)^{2}}{\left(x_{k}^{2}+y_{k}^{2}\right)\left(x_{k+1}^{2}+y_{k+1}^{2}\right)}
$$

Since $x_{k}$ and $y_{k}$ are assumed to be known, maximising $J$ is equivalent to maximising

$$
\bar{J}=\frac{\left(x_{k} y_{k+1}-x_{k+1} y_{k}\right)^{2}}{\left(x_{k+1}^{2}+y_{k+1}^{2}\right)}
$$

]$^{T}$ Taking the partial derivative of $\bar{J}$ with respect to $\gamma_{o, k}$ and substituting Eq. (13) into it yields

$$
\begin{aligned}
\frac{\partial \bar{J}}{\partial \gamma_{o, k}}= & \frac{2 V_{o, k} T_{s}\left[x_{k}\left(y_{k}-v_{t y, k}+V_{o, k} T_{s} \sin \gamma_{o, k}\right)-y_{k}\left(x_{k}-v_{t x, k}+V_{o, k} T_{s} \cos \gamma_{o, k}\right)\right]}{\left[\left(x_{k}-v_{t x, k}+V_{o, k} T_{s} \cos \gamma_{o, k}\right)^{2}+\left(y_{k}-v_{t y, k}+V_{o, k} T_{s} \sin \gamma_{o, k}\right)^{2}\right]^{2}} \\
& \times\left\{\left(x_{k} \cos \gamma_{o, k}+y_{k} \sin \gamma_{o, k}\right)\left[\left(x_{k}-v_{t x, k}+V_{o, k} T_{s} \cos \gamma_{o, k}\right)^{2}+\left(y_{k}-v_{t y, k}+V_{o, k} T_{s} \sin \gamma_{o, k}\right)^{2}\right]\right. \\
& +\left[x_{k}\left(y_{k}-v_{t y, k}+V_{o, k} T_{s} \sin \gamma_{o, k}\right)-y_{k}\left(x_{k}-v_{t x, k}+V_{o, k} T_{s} \cos \gamma_{o, k}\right)\right] \\
& \left.\times\left[\sin \gamma_{o, k}\left(x_{k}-v_{t x, k}\right)-\cos \gamma_{o, k}\left(y_{k}-v_{t y, k}\right)\right]\right\}
\end{aligned}
$$

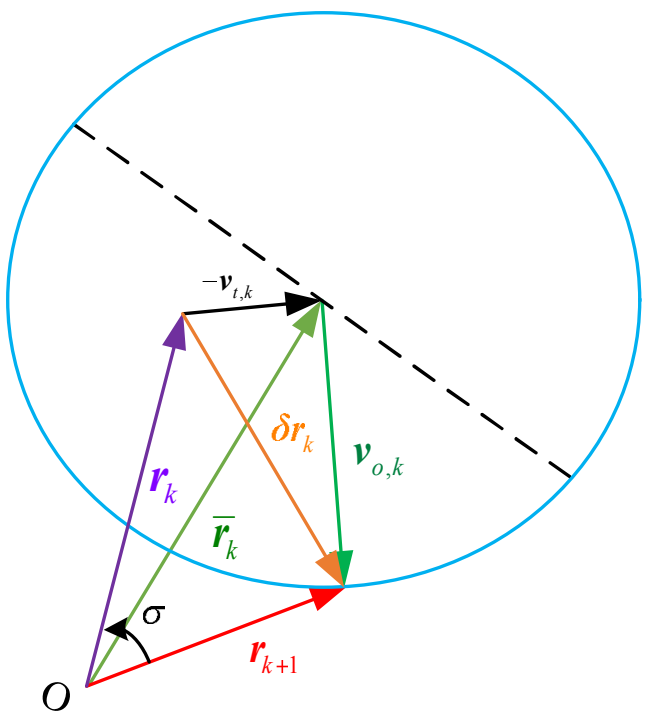

Figure 2: Geometric illustration for moving target localisation without turning rate limit in the relative frame. The blue circle determines the maximum permissible region that the observer can travel at current time step.

$$
\begin{aligned}
& \text { Solving } \partial \bar{J} / \partial \gamma_{o, k}=0 \text { gives } \\
& \begin{array}{l}
x_{k}\left(y_{k}-v_{t y, k}+V_{o, k} T_{S} \sin \gamma_{o, k}\right) \\
\quad-y_{k}\left(x_{k}-v_{t x, k}+V_{o, k} T_{s} \cos \gamma_{o, k}\right)=0
\end{array}
\end{aligned}
$$

$$
\begin{aligned}
& {\left[\left(x_{k}-v_{t x, k}\right) \cos \gamma_{o, k}+\left(y_{k}-v_{t y, k}\right) \sin \gamma_{o, k}+V_{o, k} T_{s}\right]} \\
& \quad \times\left(x_{k}^{2}+y_{k}^{2}-x_{k} v_{t x, k}-y_{k} v_{t y, k}+x_{k} V_{o, k} T_{s} \cos \gamma_{o, k}\right. \\
& \left.\quad+y_{k} V_{o, k} T_{s} \sin \gamma_{o, k}\right)=0
\end{aligned}
$$

Note that, if condition (18) is satisfied, e.g., the observer heading results in the fact that the relative manoeuvre vector $\delta \mathbf{r}_{k}$ has either the same or the opposite direction with $\mathbf{r}_{k}$, we have $\mathbf{r}_{k+1}=\lambda \mathbf{r}_{k}, \lambda \in \mathbb{R}$. This will minimises the cost function $\bar{J}$ as $\bar{J}=0$. Therefore, this should be excluded from the solution candidates. Together with the fact that the cost function $\bar{J}$ is continuous, the candidate optimal heading solutions can be obtained from condition (19). Further simplifying (19) yields

$$
\left(\mathbf{r}_{k+1}^{T} \cdot \mathbf{v}_{o, k}\right)\left(\mathbf{r}_{k}^{T} \cdot \mathbf{r}_{k+1}\right)=0
$$

Since the optimal manoeuvre satisfies the condition $\mathbf{r}_{k}^{T} \cdot \mathbf{r}_{k+1} \neq 0$, the final solution that locally maximises $\bar{J}$ is given by $\mathbf{r}_{k+1}^{T} \cdot \mathbf{v}_{o, k}=0$. This implies that the optimal observer manoeuvre vector that maximises $\sin ^{2} \sigma$ is perpendicular to next step relative position vector $\mathbf{r}_{k+1}$. Using the definitions of $\mathbf{r}_{k+1}$ and $\mathbf{v}_{o, k}$, we have

$$
a \sin \gamma_{o, k}+b \cos \gamma_{o, k}=-V_{o, k} T_{s}
$$

Solving Eq. (21) for $\gamma_{o, k}$ gives Eqs. (10) and (11), which completes the proof. 
Remark 1. Given the robot velocity $V_{o, k}$, Lemma 1 revels that there exist two locally optimal heading solutions and these two solutions are symmetric with respect to $\overline{\mathbf{r}}_{k}$. The geometric illustration of the candidate optimal solutions is presented in Fig. 3, where $-\mathbf{v}_{t, k}$ is assumed to be located on the right hand side of $\mathbf{r}_{k}$. It is easy to verify that these two candidate heading directions provide the same level of optimality if $\overline{\mathbf{r}}_{k}=\rho \mathbf{r}_{k}$ with $\rho \in \mathbb{R}$, e.g., stationary target scenario or target moves with either the same or the opposite direction as $\mathbf{r}_{k}$. Except for these particular scenarios, there exists only one global optimal solution since $\sigma_{1} \neq \sigma_{2}$.

Remark 2. Define $\gamma_{o, k}^{m}$ as the heading solution that $\mathbf{r}_{k+1}=\lambda \mathbf{r}_{k}, \lambda \in \mathbb{R}$. Then, the proof of Lemma 1 indicates that, given the robot velocity $V_{o, k}$, the cost function monotonically increases when the heading rotates from $\gamma_{o, k}^{m}$ to either $\gamma_{o, k}^{*, 1}$ or $\gamma_{o, k}^{*, 2}$ since there exists no solution of $\partial \bar{J} / \partial \gamma_{o, k}=0$ that locates between $\gamma_{o, k}^{m}$ and $\gamma_{o, k}^{*}$. This property is useful in deriving the optimal solution of the original optimisation problem.

The following lemma analyses the effect of observer's velocity on the cost function.

Lemma 2. If the heading angle satisfies condition (10) or (11), the cost function J monotonically increases with respect to the observer's velocity $V_{o, k}$.

Proof. Evaluate the partial derivative of $\bar{J}$ with respect to $V_{o, k}$ and substitute $\mathbf{r}_{k+1}^{T} \cdot \mathbf{v}_{o, k}=0$ into it gives

$$
\begin{aligned}
\frac{\partial \bar{J}}{\partial V_{o, k}}= & -2 T_{s} \frac{\left[\cos \gamma_{o, k}\left(x_{k}-v_{t x, k}+V_{o, k} T_{s} \cos \gamma_{o, k}\right)+\sin \gamma_{o, k}\left(y_{k}-v_{t y, k}+V_{o, k} T_{s} \sin \gamma_{o, k}\right)\right]}{\left(x_{k}-v_{t x, k}+V_{o, k} T_{s} \cos \gamma_{o, k}\right)^{2}+\left(y_{k}-v_{t y, k}+V_{o, k} T_{s} \sin \gamma_{o, k}\right)^{4}} \\
& \times\left(x_{k} v_{t y, k}-y_{k} v_{t x, k}+T_{s} V_{o, k} y_{k} \cos \gamma_{o, k}-T_{s} V_{o, k} x_{k} \sin \gamma_{o, k}\right)^{2} \\
& +2 T_{s} \frac{\left(y_{k} \cos \gamma_{o, k}-x_{k} \sin \gamma_{o, k}\right)\left(x_{k} v_{t y, k}-y_{k} v_{t x, k}+T_{s} V_{o, k} y_{k} \cos \gamma_{o, k}-T_{s} V_{o, k} x_{k} \sin \gamma_{o, k}\right)}{\left(x_{k}-v_{t x, k}+V_{o, k} T_{s} \cos \gamma_{o, k}\right)^{2}+\left(y_{k}-v_{t y, k}+V_{o, k} T_{s} \sin \gamma_{o, k}\right)^{2}} \\
= & 2 T_{s} \frac{\left(y_{k} \cos \gamma_{o, k}-x_{k} \sin \gamma_{o, k}\right)\left(x_{k} v_{t y, k}-y_{k} v_{t x, k}+T_{s} V_{o, k} y_{k} \cos \gamma_{o, k}-T_{s} V_{o, k} x_{k} \sin \gamma_{o, k}\right)}{\left(x_{k}-v_{t x, k}+V_{o, k} T_{s} \cos \gamma_{o, k}\right)^{2}+\left(y_{k}-v_{t y, k}+V_{o, k} T_{s} \sin \gamma_{o, k}\right)^{2}}
\end{aligned}
$$

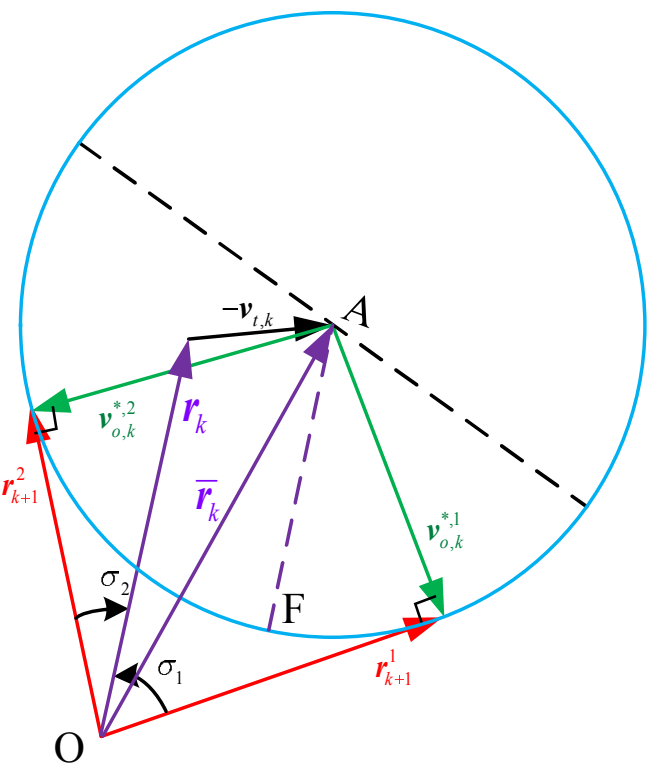

Figure 3: Geometric illustration of the candidate optimal heading solutions. $\overline{A F}$ is parallel to $\mathbf{r}_{k}$.

If $v_{o, k}^{*}$ is located on the right hand side of $\mathbf{r}_{k}$ (refer to Fig. 3), we have

$$
y_{k} \cos \gamma_{o, k}-x_{k} \sin \gamma_{o, k}>0
$$

and $\mathbf{r}_{k+1}$ is located on the right hand side of $\mathbf{r}_{k}$, e.g.,

$$
\begin{aligned}
& x_{k}\left(y_{k}-v_{t y, k}+V_{o, k} T_{s} \sin \gamma_{o, k}\right) \\
& \quad-y_{k}\left(x_{k}-v_{t x, k}+V_{o, k} T_{s} \cos \gamma_{o, k}\right)<0
\end{aligned}
$$

Substituting Eqs. (23) and (24) into Eq. (22) yields $\partial \bar{J} / \partial V_{o, k}>0$. Similarly, one can easily verify that $\partial \bar{J} / \partial V_{o, k}>0$ when $v_{o, k}^{*}$ is located on the left hand side of $\mathbf{r}_{k}$. This implies that the cost function $\bar{J}$ monotonically increases with the increase of observer's velocity $V_{o, k}$ if the heading angle satisfies condition (10) or (11), which completes the proof.

By using the results of Lemmas 1 and 2, the optimal observer manoeuvre, without any heading constraints, that maximises cost function $J$ is obtained in Theorem 1.

Theorem 1. The optimal solution of $\mathrm{CDO}_{2}$ is given by $\mathbf{v}_{o, k}^{*}=\left[V_{o, \max } T_{S} \cos \gamma_{o, k}^{*}, V_{o, \max } T_{S} \sin \gamma_{o, k}^{*}\right]^{T}$ where $\gamma_{o, k}^{*}=\max _{\gamma_{o, k} \in\left\{\gamma_{o, k}^{* 1}, \gamma_{o, k}^{*, 2}\right\}} J\left(\gamma_{o, k}\right)$ with

$$
\begin{gathered}
\gamma_{o, k}^{*, 1}=\arcsin \left(-\frac{V_{o, \max } T_{s}}{\left\|\mathbf{\mathbf { r }}_{k}\right\|}\right)-\vartheta \\
\gamma_{o, k}^{*, 2}=\pi-\arcsin \left(-\frac{V_{o, \max } T_{s}}{\left\|\mathbf{\mathbf { r }}_{k}\right\|}\right)-\vartheta
\end{gathered}
$$


Proof. From Lemmas 1 and 2, the proof of Theorem 1 is straightforward.

Corollary 1. Under the proposed algorithm without heading constraint, the relative range at next time step is given by

$$
\left\|\mathbf{r}_{k+1}\right\|=\sqrt{\left\|\overline{\mathbf{r}}_{k}\right\|^{2}-\left(V_{o, \max } T_{s}\right)^{2}}
$$

Proof. By definition of the relative range, we have

$$
\begin{aligned}
& \left\|\mathbf{r}_{k+1}\right\|^{2}=\left(x_{k}+V_{o, \max } T_{s} \cos \gamma_{o, k}^{*}-v_{t x, k}\right)^{2} \\
& +\left(y_{k}+V_{o, \max } T_{s} \sin \gamma_{o, k}^{*}-v_{t y, k}\right)^{2} \\
& =\left(x_{k}-v_{t x, k}\right)^{2}+\left(y_{k}-v_{t y, k}\right)^{2}+\left(V_{o, \max } T_{s}\right)^{2} \\
& +2 V_{o, \max } T_{s}\left[\left(x_{k}-v_{t x, k}\right) \cos \gamma_{o, k}^{*}+\left(y_{k}-v_{t y, k}\right) \sin \gamma_{o, k}^{*}\right]
\end{aligned}
$$

Substituting Eq. (21) using $V_{o, \max }$ into Eq. (28) leads to the proof of Eq. (27).

Remark 3. From Corollary 1, one can note that the relative range at next time step is influenced by the target moving speed and its direction. However, for localizing stationary target, the relative range is monotonically decreasing. This is given by the following corollary.

Corollary 2. Under the proposed algorithm without heading constraint, the relative range monotonically decreases with respect to time as

$$
\frac{d\left\|\mathbf{r}_{k}\right\|}{d t}=-\frac{V_{o, \max }^{2} T_{s}}{2\left\|\mathbf{r}_{k}\right\|}
$$

Proof. For stationary target, it is clear that $\left\|\mathbf{r}_{k}\right\|=$ $\left\|\overline{\mathbf{r}}_{k}\right\|$. Then, Eq. (27) becomes

$$
\left\|\mathbf{r}_{k+1}\right\|=\sqrt{\left\|\mathbf{r}_{k}\right\|^{2}-\left(V_{o, \max } T_{s}\right)^{2}}
$$

By definition, the rate of the relative range is determined by

$$
\frac{d\left\|\mathbf{r}_{k}\right\|}{d t}=\frac{\left\|\mathbf{r}_{k+1}\right\|-\left\|\mathbf{r}_{k}\right\|}{T_{s}}
$$

Substituting Eq. (31) into Eq. (30) and using $V_{o \text {, max }}$ yields

$$
\begin{aligned}
\frac{d\left\|\mathbf{r}_{k}\right\|}{d t} & =\frac{\sqrt{\left\|\mathbf{r}_{k}\right\|^{2}-\left(V_{o, \max } T_{s}\right)^{2}}-\left\|\mathbf{r}_{k}\right\|}{T_{S}} \\
& =\frac{\left\|\mathbf{r}_{k}\right\| \sqrt{1-\frac{\left(V_{o, \max } T_{S}\right)^{2}}{\left\|\mathbf{r}_{k}\right\|^{2}}}-\left\|\mathbf{r}_{k}\right\|}{T_{S}} \\
& \approx \frac{\left\|\mathbf{r}_{k}\right\|\left(1-\frac{1}{2} \frac{\left(V_{o, \max } T_{S}\right)^{2}}{\left\|\mathbf{r}_{k}\right\|^{2}}\right)-\left\|\mathbf{r}_{k}\right\|}{T_{S}} \\
& =-\frac{V_{o, \max }^{2} T_{S}}{2\left\|\mathbf{r}_{k}\right\|}
\end{aligned}
$$

\subsection{Optimal Solution with Heading Constraints}

As discussed in Remark 1, if the target is stationary or moves with either the same or the opposite direction as $\mathbf{r}_{k}$, the candidate heading solutions, given by Lemma 1, provide the same level of optimality. This might generate multiple candidate trajectories and zigzag heading change in practice. Therefore, it is necessary to choose only one heading solution that satisfies the practical heading change constraint when implementing Theorem 1 in real applications. For this reason, this subsection will propose the optimal solution of the constrained problem, e.g., $\mathbf{C D O}_{1}$.

Let $\gamma_{o, k-1}^{*}$ be the optimal heading angle at the previous time step. Without loss of generality, assume that $\gamma_{o, k}^{*, 1}$ is closer to $\gamma_{o, k-1}^{*}$ than $\gamma_{o, k}^{*, 2}$, e.g., $\left|\gamma_{o, k}^{*, 1}-\gamma_{o, k-1}^{*}\right| \leq\left|\gamma_{o, k}^{*, 2}-\gamma_{o, k-1}^{*}\right| \cdot \quad$ Denote $\Xi$ as the permissible heading angle set, e.g., $\Xi \triangleq$ $\left\{\gamma_{o, k} \mid \gamma_{o, k-1}^{*}-\gamma_{\max } \leq \gamma_{o, k} \leq \gamma_{o, k-1}^{*}+\gamma_{\max }\right\}$, then, the solution of $\mathbf{C D O}_{1}$ is determined as follows.

Condition 1: $\gamma_{o, k}^{*, 1} \in \Xi$ and $\gamma_{o, k}^{*, 2} \in \Xi$. Under this condition, the optimal heading angle is obviously given by $\gamma_{o, k}^{*}=\max _{\gamma_{o, k} \in\left\{\gamma_{o, k}^{* 1}, \gamma_{o, k}^{*, 2}\right\}} J\left(\gamma_{o, k}\right)$. If these two candidate heading solutions provide the same level of optimality, the heading direction, that is closer to previous time step heading, is determined as the current heading direction to generate a consistent and unique solution, thus avoiding large heading change. With this in mind, the optimal heading angle is given by

$$
\gamma_{o, k}^{*}=\left\{\begin{array}{cl}
\gamma_{o, k}^{*, 1}, \quad \text { if } J\left(\gamma_{o, k}^{*, 1}\right)=J\left(\gamma_{o, k}^{*, 2}\right) \\
\max _{\gamma_{o, k} \in\left\{\gamma_{o, k}^{* 1}, \gamma_{o, k}^{*, 2}\right\}} J\left(\gamma_{o, k}\right), & \text { otherwise }
\end{array}\right.
$$

Condition 2: $\gamma_{o, k}^{*, 1} \in \Xi$ and $\gamma_{o, k}^{*, 2} \notin \Xi$. Since the cost function monotonically increases when the heading rotates from $\gamma_{o, k}^{m}$ to either $\gamma_{o, k}^{*, 1}$ or $\gamma_{o, k}^{*, 2}$, the optimal heading solution is given by

$$
\gamma_{o, k}^{*}=\max _{\gamma_{o, k} \in\left\{\gamma_{o, k}^{* 1,}, \bar{\gamma}_{o, k}^{* 1,}, \bar{\gamma}_{o, k}^{*, 2}\right\}} J\left(\gamma_{o, k}\right)
$$

where $\bar{\gamma}_{o, k}^{*, 1}=\gamma_{o, k-1}^{*}-\gamma_{\max }$ and $\bar{\gamma}_{o, k}^{*, 2}=\gamma_{o, k-1}^{*}+\gamma_{\max }$.

Condition 3: $\gamma_{o, k}^{*, 1} \notin \Xi$ and $\gamma_{o, k}^{*, 2} \notin \Xi$. Under this condition, the optimal heading solution is obviously located at the boundary of $\Xi$ as

$$
\gamma_{o, k}^{*}=\max _{\gamma_{o, k} \in\left\{\bar{\gamma}_{o, k}^{* 1}, \bar{\gamma}_{o, k}^{*, 2}\right\}} J\left(\gamma_{o, k}\right)
$$


Remark 4. Note that when $\gamma_{o, k}^{*, 2}$ is closer to $\gamma_{o, k-1}^{*}$ than $\gamma_{o, k}^{*, 1}$, i.e., $\left|\gamma_{o, k}^{*, 1}-\gamma_{o, k-1}^{*}\right|>\left|\gamma_{o, k}^{*, 2}-\gamma_{o, k-1}^{*}\right|$, similar results can also be obtained.

Remark 5. It is worthy pointing out that the observer, under the proposed algorithm, will never collide with the target since the minimum achievable relative range is limited by the turning rate constraint.

By summarizing the aforementioned three conditions, the proposed active sensing strategy for target localisation using range-only measurements is summarized in Algorithm 1.

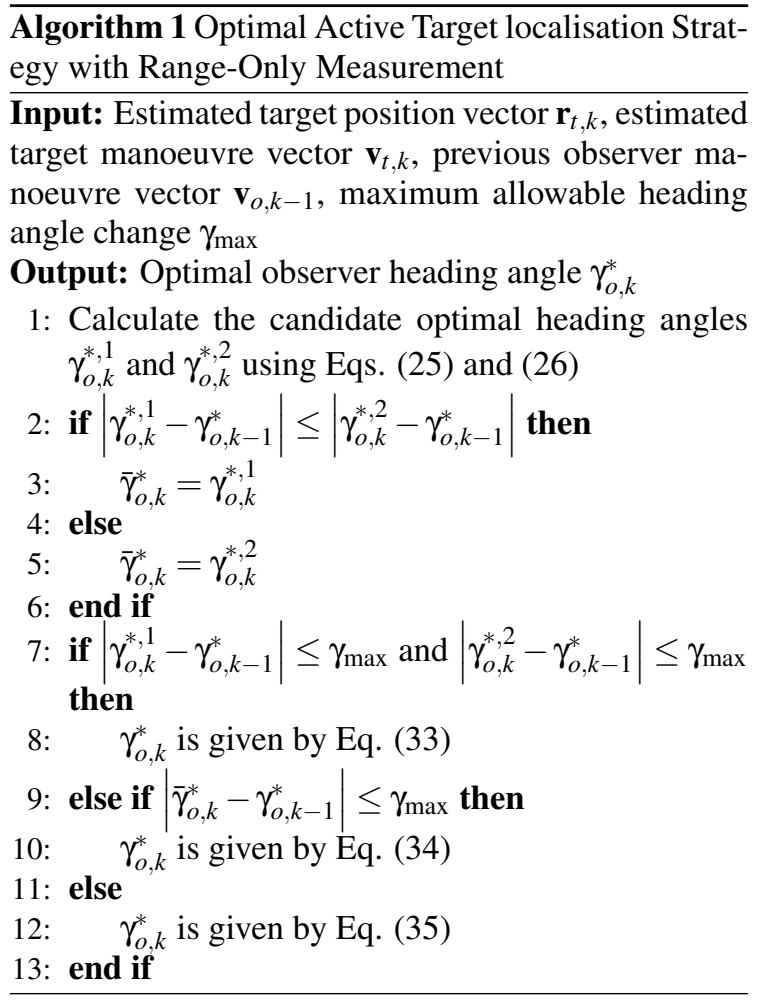

\section{Observability Analysis}

The baseline assumption that we utilised in deriving the optimal heading solution is that current target position and velocity vector are available to the observer. This information, however, is extracted from an estimator in practical applications. For this reason, it is necessary to analyse system observability since $\mathbf{r}_{k}$ is estimable only when target is observable. The results are presented in the following proposition.

Proposition 1. Under the proposed algorithm, the target is always observable.
Proof. The necessary and sufficient condition to guarantee system observability for localising nonmanoeuvring target with range-only measurement is given by (Song, 1999)

$$
\mathbf{r}(t) \neq\left[\begin{array}{l}
a_{11}+a_{12} \Delta t \\
a_{21}+a_{22} \Delta t
\end{array}\right]
$$

for some $t \in\left(t_{0}, t_{f}\right]$ with $t_{0}$ being the initial time, where $\Delta t=t-t_{0}$. The coefficients $a_{i j}$ in Eq. (36) are arbitrary constants but not all zero.

If the target is unobservable, if follows from condition (36) that there exists a line such that the relative position always locates on the line with equal length between two consecutive time steps, e.g., $\delta \mathbf{r}_{i}=\delta \mathbf{r}_{j}$, $\forall i \neq j$. This means that observer manoeuvre that changes either the magnitude of the relative velocity or the direction of the relative velocity is required for range-only target localisation scenario. From previous analysis, we know that the proposed algorithm leverages the maximum observer velocity and generates a unique solution that forces the relative position vector rotate either clockwise or anti-clockwise around the target. Therefore, the direction of the relative velocity changes at every time instant, which implies that there exists some $t \in\left(t_{k}, t_{f}\right]$ such that condition (36) is satisfied $\forall t_{k}$. This means that target is always observable under the proposed approach.

\section{Simulation Studies}

In this section, estimator-in-the-loop simulations are performed to validate the proposed optimal sensing strategy. The initial position of the robot is $(20 \mathrm{~m}, 50 \mathrm{~m})$ with initial heading $0^{\circ}$. The maximum permissible velocity of the robot is set as $V_{o, \max }=$ $20 \mathrm{~m} / \mathrm{s}$. The turning rate of the robot is constrained by $\omega_{\max }=1 \mathrm{rad} / \mathrm{s}$ and the sampling time is set as $T_{s}=0.1 \mathrm{~s}$. The target initially locates at $(0 \mathrm{~m}, 0 \mathrm{~m})$ and moves in a straight line with constant velocity $V_{t}=$ $5.83 \mathrm{~m} / \mathrm{s}$ as well as constant heading $\gamma_{t}=0.54 \mathrm{rad}$. To implement the proposed algorithm, the required information on target position and velocity are extracted from the well-known extended Kalman filter in conjunction with a constant velocity model. For validation, the numerical optimal solution of $\mathbf{C D O}_{1}$ obtained from Particle Swarm optimisation algorithm is also presented.

Figure 4 presents the moving trajectories of the target and the observer. From this figure, one can note that the proposed algorithm forces the relative position vector rotate clockwise in the considered scenario for target localisation. The determinant of FIM is shown in Fig. 5, demonstrating that $\operatorname{det}(\mathbf{F I M})$ increases monotonically with respect to time. Since 


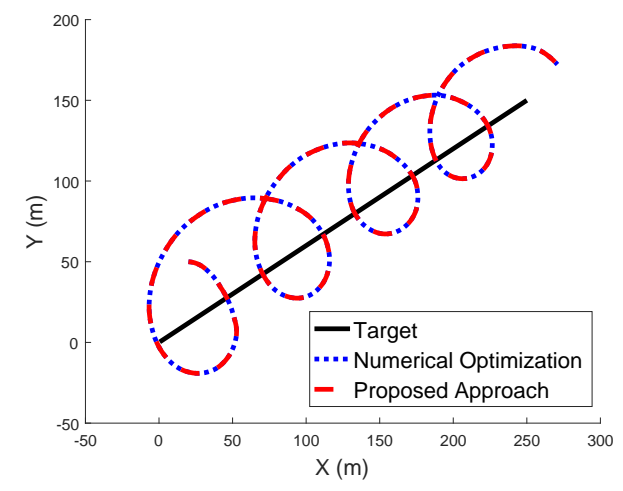

Figure 4: Moving trajectories.

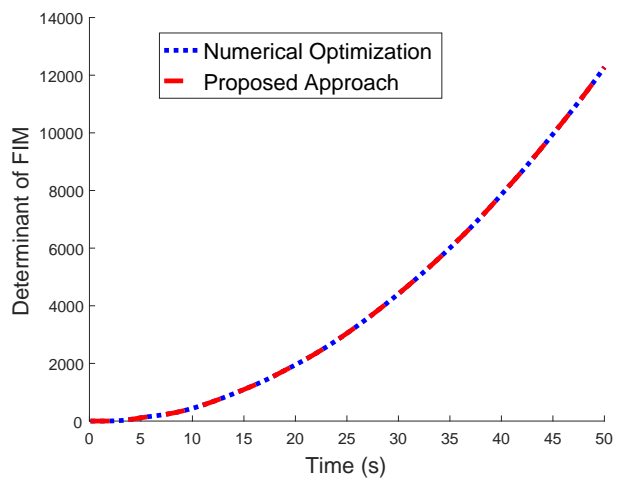

Figure 5: Determinant of FIM.

$\operatorname{det}(\mathbf{F I M})$ quantifies the volume of the estimation error uncertainty ellipsoid, target localisation performance can be improved using the proposed active sensing algorithm. Figure 6 provides the history of observer's heading rate, which reveals that the physical turning rate constraint is satisfied. Furthermore, from the obtained results, we can clearly observe that the proposed analytical solution coincides with the numerical optimal solutions, demonstrating the effectiveness of the proposed algorithm. Future work includes extending the proposed approach to heterogeneous sensors.

\section{Conclusions}

The problem of active target localisation using range-only measurements is studied in this paper. By leveraging the determinant of one-step ahead FIM as the cost function and heading angle command as the control input, the discrete-time optimal heading is derived analytically to minimise target localisation error. Both velocity and turning rate limits are considered in the proposed optimisation approach. Numerical sim-

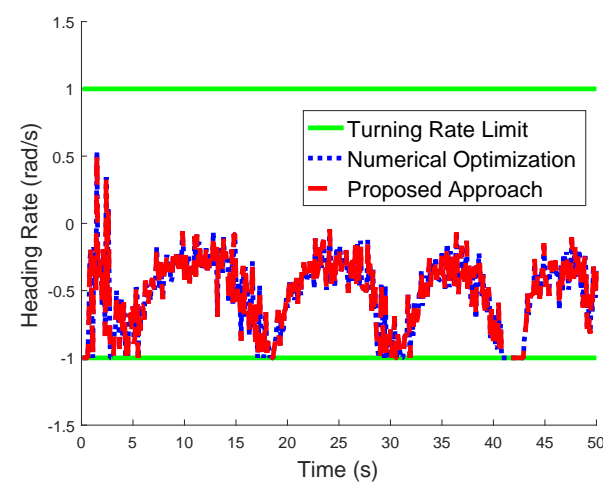

Figure 6: Turning rate.

ulations are performed to validate the analytical finds.

\section{REFERENCES}

Atanasov, N., Le Ny, J., Daniilidis, K., and Pappas, G. J. (2014). Information acquisition with sensing robots: Algorithms and error bounds. In Robotics and Automation (ICRA), 2014 IEEE International Conference on, pages 6447-6454. IEEE.

Bajcsy, R., Aloimonos, Y., and Tsotsos, J. K. (2018). Revisiting active perception. Autonomous Robots, 42(2):177196.

Bishop, A. N., Fidan, B., Anderson, B. D., Doğançay, K., and Pathirana, P. N. (2010). Optimality analysis of sensor-target localization geometries. Automatica, 46(3):479-492.

Cao, Y. (2015). UAV circumnavigating an unknown target under a GPS-denied environment with range-only measurements. Automatica, 55:150-158.

He, S., Shin, H.-S., and Tsourdos, A. (2019). Trajectory optimisation for target localisation with bearing-only measurement. IEEE Transactions on Robotics.

Logothetis, A., Isaksson, A., and Evans, R. J. (1997). An information theoretic approach to observer path design for bearings-only tracking. In IEEE Conference on Decision and Control, volume 4, pages 3132-3137. IEEE.

MartíNez, S. and Bullo, F. (2006). Optimal sensor placement and motion coordination for target tracking. Automatica, 42(4):661-668.

Matveev, A. S. and Semakova, A. A. (2017). Range-only based $3 \mathrm{~d}$ circumnavigation of multiple moving targets by a non-holonomic mobile robot. IEEE Transactions on Automatic Control.

Matveev, A. S., Semakova, A. A., and Savkin, A. V. (2016) Range-only based circumnavigation of a group of moving targets by a non-holonomic mobile robot. Automatica, 65:76-89. 
Matveev, A. S., Teimoori, H., and Savkin, A. V. (2011). Range-only measurements based target following for wheeled mobile robots. Automatica, 47(1):177-184.

Salaris, P., Spica, R., Giordano, P. R., and Rives, P. (2017). Online optimal active sensing control. In Robotics and Automation (ICRA), 2017 IEEE International Conference on, pages 672-678. IEEE.

Song, T. L. (1999). Observability of target tracking with range-only measurements. IEEE Journal of Oceanic Engineering, 24(3):383-387.

Taylor, J. (1979). The cramer-rao estimation error lower bound computation for deterministic nonlinear systems. IEEE Transactions on Automatic Control, 24(2):343344.

Tokekar, P., Vander Hook, J., and Isler, V. (2011). Active target localization for bearing based robotic telemetry. In Intelligent Robots and Systems (IROS), 2011 IEEE/RSJ International Conference on, pages 488-493. IEEE.

Yang, Z., Shi, X., and Chen, J. (2014). Optimal coordination of mobile sensors for target tracking under additive and multiplicative noises. IEEE Transactions on Industrial Electronics, 61(7):3459-3468.

Zhou, K., Roumeliotis, S. I., et al. (2011). Multirobot active target tracking with combinations of relative observations. IEEE Transactions on Robotics, 27(4):678-695. 
2019-07-31

\section{Optimal active target localisation strategy with range-only measurements}

He, Shaoming

INSTICC

He S, Shin H-S, Tsourdos A. (2019) Optimal active target localisation strategy with range-only measurements. In: ICINCO 2019: 16th International Conference on Informatics in Control, Automation and Robotics, 29-31 July 2019, Prague, Czech Republic http://www.icinco.org/?y=2019

Downloaded from Cranfield Library Services E-Repository 